\section{Efficacy of a Washer-Disinfector in Eliminating Healthcare-Associated Pathogens from Surgical Instruments}

\author{
William A. Rutala, $\mathrm{PhD}, \mathrm{MPH} ;{ }^{1,2}$ \\ Maria F. Gergen, MT (ASCP); ${ }^{1}$ \\ David J. Weber, MD, MPH ${ }^{1,2}$
}

\section{METHODS}

\section{Description of Washer-Disinfector}

The Steris Reliance 777 automated multichamber washerdisinfector (Steris) was used. The preprogrammed cycle included 5 phases: (1) prewash: enzyme (Klenzyme Enzymatic Presoak and Cleaner, Steris) is circulated over the load for minimally 1 minute; (2) wash: detergent (Mon-Klenz Neutral Cleaner, Steris) solution $\left(150^{\circ}-180^{\circ} \mathrm{F}\right)$ is sprayed over the load for minimally 4 minutes; (3) ultrasonic cleaning: the basket is lowered into the ultrasonic-cleaning tank with solution at $150^{\circ}-180^{\circ} \mathrm{F}$ for 4 minutes; (4) thermal and instrument lubrication: hot water $\left(180^{\circ}-200^{\circ} \mathrm{F}\right)$ is sprayed over the load for 1 minute, then instrument lubricant (Hinge-Free Instrument Lubricant, Steris) is added to the water and sprayed over the load; and (5) drying: the blower runs for at least 4 minutes, resulting in a drying temperature of $240^{\circ} \mathrm{F}$. The Biomedical Engineering staff verified that the washer-disinfector was functioning properly before experimentation and assisted in disabling the washer-disinfector.

\section{Test Organisms}

Test bacterial suspensions (ie, vancomycin-resistant Enterococcus [VRE], methicillin-resistant Staphylococcus aureus [MRSA], and Pseudomonas aeruginosa) were prepared fresh daily by plating each organism to sheep blood agar (SBA; Remel) and incubating at $37^{\circ} \mathrm{C}$ for $\sim 18$ hours before each run. Mycobacterium terrae (frozen culture) and Geobacillus stearothermophilus (refrigerated) suspensions were inoculated into Trypticase soy broth (TSB; Remel) prior to use. Immediately before each run, $0.5-\mathrm{McF}$ arland standard suspensions were prepared from these plates, separately for each organism in TSB. The following test organisms were used: MRSA (ATCC 29213), VRE (ATCC 51299), P. aeruginosa (ATCC 27853), M. terrae (Wayne ATCC 15755), and G. stearothermophilus spores (Charles Roberts, Advanced Sterilization Products).

\section{Inoculation of the Test Instruments}

Four instruments were inoculated with the test organisms: a Stille-Luer rongeur, a Kerrison rongeur, a vaginal speculum, and a Kocher clamp. In the initial series of experiments (Table 1 ), the 4 instruments were inoculated with $10 \mu \mathrm{L}$ of the test organisms in TSB (ie, $\sim 3 \times 10^{7}$ MRSA, $\sim 3 \times 10^{7} \mathrm{VRE}$, $\sim 2 \times 10^{7} \mathrm{P}$. aeruginosa, $\sim 1 \times 10^{8} \mathrm{M}$. terrae, and at least $5 \times$ $10^{6}$ G. stearothermophilus spores). The inocula were placed on an exposed surface of the instrument and allowed to air dry in a biological safety cabinet for 1 hour. After drying, each instrument was transported to Central Processing and processed in the washer-disinfector. After the cycle was complete, the instruments were aseptically placed in peel packs for transport to the laboratory. Each instrument was then immersed 
TABLE 1. Elimination of Microbial Contamination on Exposed Surfaces of Experimentally Contaminated Surgical Instruments by Use of a Washer-Disinfector

\begin{tabular}{lccc}
\hline Washer-disinfector conditions, organism & $\begin{array}{c}\text { Mean inoculum, } \\
\log _{10}{ }^{\mathrm{a}}\end{array}$ & $\begin{array}{c}\text { Mean reduction, } \\
\log _{10}{ }^{\mathrm{b}}\end{array}$ & $\begin{array}{c}\text { Proportion } \\
\text { of positive } \\
\text { instruments/replicates }\end{array}$ \\
\hline Routine & & & \\
Methicillin-resistant Staphylococcus aureus & 7.4 & $>7.4^{\mathrm{d}}$ & $0 / 8$ \\
Vancomycin-resistant Enterococcus & 7.4 & $>7.4^{\mathrm{d}}$ & $0 / 8$ \\
Pseudomonas aeruginosa & 7.3 & $>7.3^{\mathrm{d}}$ & $0 / 8$ \\
Mycobacterium terrae & 8.1 & 7.8 & $2 / 8$ \\
Geobacillus stearothermophilus spores & 6.7 & 4.8 & $11 / 14$ \\
No enzymatic cleaner/detergent & & & $0 / 10$ \\
Vancomycin-resistant Enterococcus & 7.4 & $>7.4^{\mathrm{d}}$ & $8 / 10$ \\
G. stearothermophilus spores & 6.9 & 5.5 &
\end{tabular}

a Positive control was performed by bioburden extraction from surgical instruments that were inoculated under the same conditions as the surgical instruments but not processed in the washer-disinfector.

${ }^{b} \log _{10}$ reductions were calculated from the differences between the recovered counts on the control (unprocessed) and test (processed) items.

c All test conditions (ie, washer-disinfector [routine or no enzymatic cleaner/detergent] with test microorganisms and surgical instruments) were tested in 2-5 independent washer-disinfector runs.

d Complete microbial elimination achieved.

fully in sterile TSB to allow adequate contact with all surfaces (300-400 mL) and shaken on a clinical rotator for 1 hour at $110 \mathrm{rpm}$. After shaking, samples $\left(10^{-1}, 10^{-2}\right.$ dilutions) were collected for quantification of the test organism. These samples were plated to SBA or 7H11 (for M. terrae) in duplicate via a spread plate and incubated at $37^{\circ} \mathrm{C}\left(53^{\circ} \mathrm{C}\right.$ for $\mathrm{G}$. stearothermophilus) for 48 hours ( 4 weeks for $M$. terrae). The remaining amount was filtered $(0.22-\mu \mathrm{m}$ mesh, Fisher Scientific) and the filter plated to SBA (or 7 H11 for M. terrae) and incubated in the same manner. All growth was quantitated and identified.

The second set of experiments were done identically, except that the inocula were placed in the hinged area of a medical device (or the screw threads of the vaginal speculum), and then the hinged instruments were closed (normally open to allow contact with the detergent solution) or the thumb screw of the vaginal speculum was tightened.

\section{RES U L T S}

The washer-disinfector tested was extremely effective $(>7$ $\log _{10}$ reduction) in eliminating contamination with both the exposed and the nonexposed test bacteria (MRSA, VRE, $P$. aeruginosa, and M. terrae; Table 1). The exposed G. stearothermophilus spores were substantially reduced $\left(\sim 5-\log _{10}\right.$ reduction), but the nonexposed or "hidden" spores were not eliminated (Table 2). The washer-disinfector remained effective in eliminating or dramatically reducing the level of contaminating bacteria and spores in the absence of the detergent and enzymatic cleaner (Tables 1,2), except when the spores were "hidden."

\section{I S C US S I O N}

These experiments demonstrated the extreme effectiveness of the washer-disinfector in eliminating or reducing high num- bers of pathogenic bacteria and spores from stainless steel surgical instruments. We found that disabling the washerdisinfector by preventing the introduction of the detergent and the enzymatic cleaner did not alter the effectiveness of the washer-disinfector against bacteria, as high numbers of clinically relevant bacteria were completely eliminated (Table 1). These data are similar to those of Nicolaos et $\mathrm{al}^{4}{ }^{4}$ who found an $8-\log _{10}$ reduction of Enterococcus on inoculated screws. However, design features such as crevices, hinges, and covered surfaces (eg, screw threads covered by thumb screw) of surgical instruments protect the inocula (ie, spores) from the forced flow of liquids and inhibited elimination (Table 2). Thus, fluids under pressure (water with/without cleaners), ultrasonic cleaning, and thermal inactivation $\left(150^{\circ}-240^{\circ} \mathrm{F}\right)$ are sufficient to eliminate microorganisms but not spores that are "hidden" and relatively resistant to thermal inactivation. Fluids under pressure physically remove microorganisms, while ultrasonic cleaning removes soil and microorganisms by cavitation and implosion, in which waves of acoustic energy are propagated in aqueous solutions to disrupt the bonds that hold particulate matter to surfaces. G. stearothermophilus spores, which are relatively resistant to heat, survived the washer-disinfector process when not removed by fluidics or ultrasonic cleaning. It should be noted that the inocula used in these experiments far exceed the microbial load on used surgical instruments, as previous research has demonstrated that surgical instruments are most commonly contaminated with fewer than 100 vegetative bacteria. ${ }^{6,7}$

These experiments also showed that thermal inactivation, fluids, and ultrasonic energy were more important than the cleaning agents in eliminating microorganisms, as effective elimination occurred in the absence of enzymatic cleaners and detergents. We continue to recommend the use of enzymatic cleaners and detergents to eliminate proteinaceous materials if 
TA B LE 2. Elimination of Microbial Contamination on Nonexposed Surfaces of Experimentally Contaminated Surgical Instruments (nonexposed) by Use of a Washer-Disinfector

\begin{tabular}{lccc}
\hline Washer-disinfector conditions, organism & $\begin{array}{c}\text { Mean inoculum, } \\
\log _{10}{ }^{\mathrm{a}}\end{array}$ & $\begin{array}{c}\text { Mean reduction, } \\
\log _{10}{ }^{\mathrm{b}}\end{array}$ & $\begin{array}{c}\text { Proportion } \\
\text { of positive } \\
\text { instruments/replicates }\end{array}$ \\
\hline Routine & & & \\
Methicillin-resistant Staphylococcus aureus & 7.4 & $>7.4^{\mathrm{d}}$ & $0 / 8$ \\
Vancomycin-resistant Enterococcus & 7.5 & $>7.5^{\mathrm{d}}$ & $0 / 8$ \\
Pseudomonas aeruginosa & 7.3 & $>7.3^{\mathrm{d}}$ & $0 / 8$ \\
Mycobacterium terrae & 8.1 & 7.6 & $6 / 8$ \\
Geobacillus stearothermophilus spores & 6.9 & 1.6 & $12 / 12$ \\
No enzymatic cleaner/detergent & & & $0 / 10$ \\
Vancomycin-resistant Enterococcus & 7.4 & $>7.4^{\mathrm{d}}$ & $10 / 10$ \\
G. stearothermophilus spores & 6.9 & 1.6 & \\
\hline
\end{tabular}

a Positive control was performed by bioburden extraction from surgical instruments, which were inoculated under the same conditions as the surgical instruments but not processed in the washer-disinfector.

${ }^{\mathrm{b}} \log _{10}$ reductions were calculated from the differences between the recovered counts on the control (unprocessed) and test (processed) items.

c All test conditions (ie, washer-disinfector [routine or no enzymatic cleaner/detergent] with test microorganisms and surgical instruments) were tested in 2-5 independent washer-disinfector runs.

d Complete microbial elimination achieved.

instruments are not adequately precleaned. While cleaning is critical in eliminating microbial contamination from instruments, it is important to note that there are no US Food and Drug Administration-cleared commercially available methods for rapid detection of soil components (eg, protein level of $<6.4 \mu \mathrm{g} / \mathrm{cm}^{2}$ ) that can be used by hospitals to monitor cleaning effectiveness.

In conclusion, washer-disinfectors are extremely effective at eliminating microorganisms from medical and surgical instruments. The elimination of microorganisms occurs by removal (eg, fluidics, detergents, and ultrasound) and thermal inactivation associated with the high-temperature wash-and-dry cycle.

\section{ACKNOWLEDGMENTS}

We acknowledge Mr. Tony Ma and Mr. Dennis Hildreth, of Medical Engineering, and Ms. Betty Twamley, of Central Processing, for their assistance with verifying the operation of the washer-disinfector and facilitating this investigation.

Potential conflicts of interest. W.A.R. reports that he is a consultant to Clorox and Advanced Sterilization Products; D.J.W. reports that he is a consultant to Johnson \& Johnson and Clorox; M.F.G. reports no conflicts of interest relevant to this article. All authors submitted the ICMJE Form for Disclosure of Potential Conflicts of Interest, and the conflicts that the editors consider relevant to this article are disclosed here.

Affiliations: 1. Hospital Epidemiology, University of North Carolina Health Care, Chapel Hill, North Carolina; 2. Division of Infectious Diseases,
University of North Carolina School of Medicine, Chapel Hill, North Carolina.

Address correspondence to William Rutala, PhD, MPH, 130 Mason Farm Road, Bioinformatics, Chapel Hill, NC 27599-7030 (brutala@unch.unc.edu). Received November 26, 2013; accepted March 1, 2014; electronically published May 20, 2014.

(C) 2014 by The Society for Healthcare Epidemiology of America. All rights reserved. 0899-823X/2014/3507-0017\$15.00. DOI: 10.1086/676867

\section{REFERENCES}

1. Jacobs P. Cleaning: principles, methods and benefits. In: Rutala WA, ed. Disinfection, sterilization, and antisepsis in healthcare. Champlain, NY: Polyscience, 1998:165-181.

2. Alfa MJ, Olson N, DeGagne P. Automated washing with the Reliance endoscope processing system and its equivalence to optimal manual cleaning. Am J Infect Control 2006;34(9):561-570.

3. Alfa MJ, Degagne P, Olson N. Worst-case soiling levels for patientused flexible endoscopes before and after cleaning. Am J Infect Control 1999;27(5):392-401.

4. Nicolaos G, Lescoat A, Blouin P, et al. Comparison of the efficiency of cleaning, disinfection and drying of three washer-disinfectors: Steris T21, Steris T840 and Getinge 9128. J Hosp Infect 2008;68(3):278-280.

5. Alfa MJ, Olson N, Al-Fadhaly A. Cleaning efficacy of medical device washers in North American healthcare facilities. J Hosp Infect 2010;74(2):168-177.

6. Rutala WA, Gergen MF, Jones JF, Weber DJ. Levels of microbial contamination on surgical instruments. Am J Infect Control 1998; 26(2):143-145.

7. Nystrom B. Disinfection of surgical instruments. J Hosp Infect 1981;2(4):363-368. 